Check for updates

Cite this: RSC Adv., 2019, 9, 7757

Received 28th November 2018

Accepted 1st March 2019

DOI: 10.1039/c8ra09789e

rsc.li/rsc-advances

\section{Fragment-centric topographic mapping method guides the understanding of ABCG2-inhibitor interactions $\uparrow$}

\author{
Yao Wu, ${ }^{a}$ Xin-Ying Gao, ${ }^{a}$ Xin-Hui Chen, ${ }^{a}$ Shao-Long Zhang, ${ }^{b}$ Wen-Juan Wang, ${ }^{a}$ \\ Xie-Huang Sheng (iD *a and De-Zhan Chen (D) *a
}

\begin{abstract}
Understanding protein-ligand interactions is crucial to drug discovery and design. However, it would be extremely difficult for the proteins which only have one available apo structure but multiple binding sites. To address this constraint, a fragment-centric topographic mapping method (AlphaSpace software) was employed to map out concave interaction pockets at the assigned protein region. These pockets are used as complementary spaces to screen the known inhibitors for this specific binding site and to guide the molecular docking pose selection as well as protein-ligand interaction analysis. By mapping the shape of central cavity surface, we have tested the strategy against a multi-drug resistant transmembrane protein-ABCG2 to assist in generating a pharmacophore model for its inhibitors that is based on the structure of apo. Classical molecular simulation and accelerated molecular simulation are used to verify the accuracy of inhibitor screening and binding pose selection. Our study not only has gained insight for the development of novel specific ABCG2 inhibitors, but also has provided a general strategy in describing protein-ligand interactions.
\end{abstract}

\section{Introduction}

Protein-ligand interactions play a vital role in all biological processes ranging from metabolic enzyme catalysis to regulation of complex signalling cascades. ${ }^{1}$ Knowledge on the molecular details of these interactions is crucial to complete the understanding of the biological system and to discover drugs for the treatment of diseases. Protein-ligand interactions mainly concern the binding affinity, steric complementarity of the surface and pharmacophoric patterns of the compound to the target protein. ${ }^{2}$ Heretofore, crystal structures of proteinligand complexes are the best models to provide a detailed view of their spatial arrangements and interactions. ${ }^{3}$ However, there are still many proteins that only have one available apo structures in PDB database.

Molecular docking is an effective tool in predicting the structures of protein-ligand complexes as well as studying the protein-ligand interactions, and evaluating the binding affinities of such complexes. ${ }^{4}$ A typical docking program implements

${ }^{a}$ College of Chemistry, Chemical Engineering and Materials Science, Collaborative Innovation Centre of Functionalized Probes for Chemical Imaging in Universities of Shandong, Key Laboratory of Molecular and Nano Probes, Ministry of Education, Shandong Provincial Key Laboratory of Clean Production of Fine Chemicals, Shandong Normal University, Jinan 250014, P. R. China

${ }^{b}$ College of Physics and Electronics, Shandong Normal University, Jinan 250014, P. R. China.E-mail: shengxiehuang@sdnu.edu.cn; dchen@sdnu.edu.cn

$\dagger$ Electronic supplementary information (ESI) available: Additional simulated and computational results involved in this study. See DOI: $10.1039 / \mathrm{c} 8 \mathrm{ra09789e}$ a sampling algorithm to generate possible binding poses and a scoring function of binding affinity estimation. ${ }^{5}$ Modern docking tools such as AutoDock Vina ${ }^{6}$ and glide ${ }^{7}$ are currently capable of generating near-native poses with a re-docking success rate at over $50 \%$ on three diverse benchmarks. ${ }^{8}$ However, the near-native poses are often not the lowest binding energy ones. Thus, it is still a challenge for pharmacologists to identify the correct binding pose from the poses library., ${ }^{\mathbf{9 , 1 0}}$ Understanding of protein-ligand interactions is directly determined by the accurate selection of the binding pose. ${ }^{\mathbf{1 1 , 1 2}}$ It would produce an unreliable result of protein-ligand interaction and greatly hinder drug development if an incorrect one was selected. Under this circumstance, a reliable theoretical method is an extraordinarily urgent need to improve the ability of selecting correct docking pose and to better understand the interaction between known inhibitors with protein in apo structure.

Human ABCG2 is one of the three major human ATP binding cassette $(\mathrm{ABC})$ transporters (the other two are P-glycoprotein and MRP1). ${ }^{13}$ It facilitates the efflux of a broad range of anticancer drugs, ${ }^{\mathbf{1 4}}$ decreases the intracellular accumulation of cytotoxic drug and impairs the success of chemotherapeutic regimens. ${ }^{15-17}$ Over-expression of ABCG2 has been frequently found in various drug-selected cancer cell lines. It contributes to clinical MDR of solid tumours and hematopoietic malignancies (Fig. 1). ${ }^{18-20}$ ABCG2 transports a structurally diverse array of substrates, some shared with P-glycoprotein and MRP1 while others are ABCG2 specific, ${ }^{21}$ including topoisomerase 


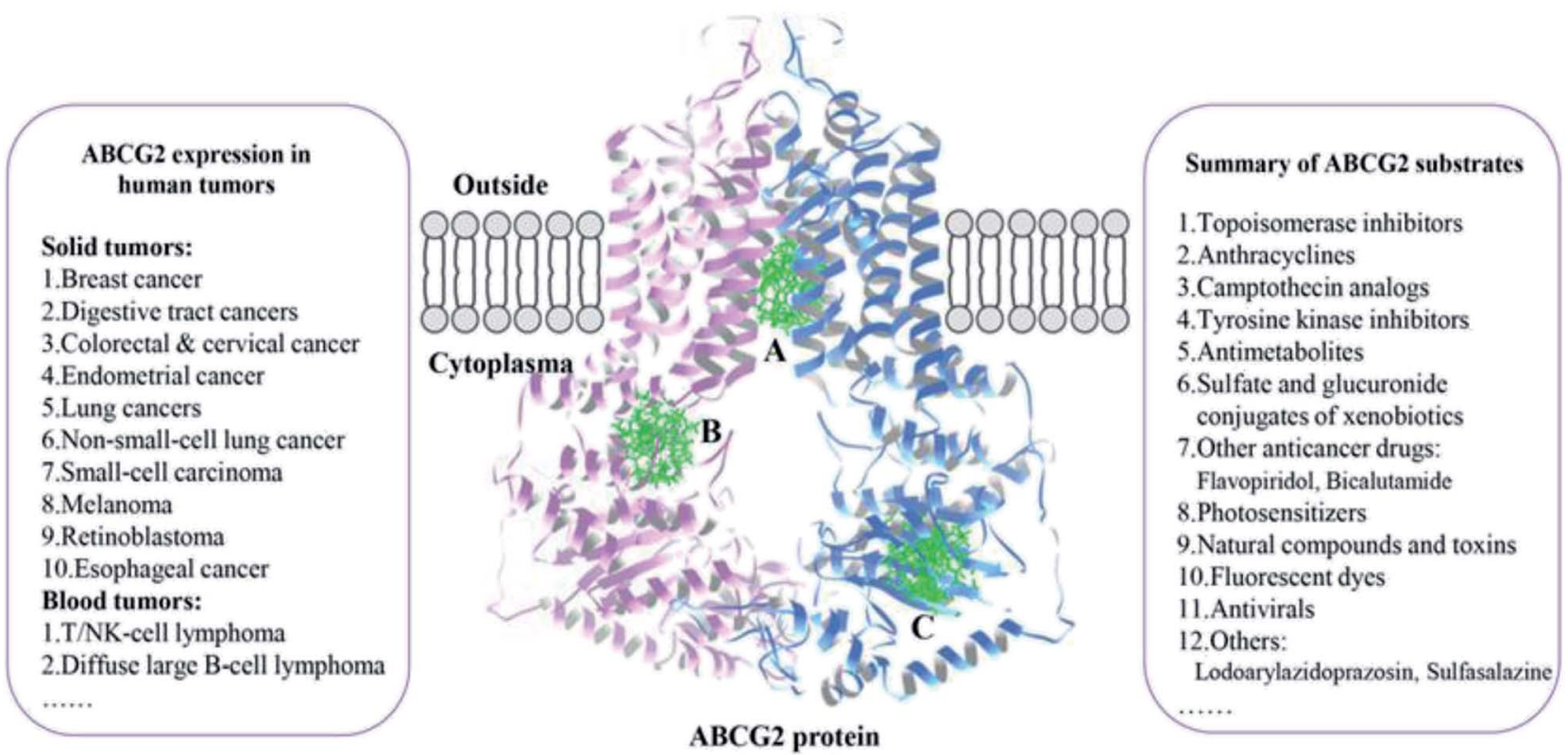

Fig. 1 The human tumors caused by ABCG2 over-expression and summary of ABCG2 substrates are listed, and the candidate binding sites of $A B C G 2$ are also marked in this figure.

inhibitors, anthracyclines, camptothecin analogues, tyrosine kinase inhibitors, antimetabolites, non-nucleoside reverse transcriptase inhibitors and protease inhibitors (Fig. 1). ${ }^{22}$

Owing to their potential values as reversal agents for the treatment of cancer chemotherapy, extensive studies have been made to the development of specific inhibitors against human ABCG2. Fumitremorgin C (FTC) was the first ABCG2 inhibitor to be described in 1998, it reversed chemo-resistance of colon carcinoma to MTX. ${ }^{23}$ Since then, nearly a hundred agents have been described that inhibit in vitro the action of ABCG2. ${ }^{22}$ Unfortunately, none of these compounds has been used clinically. ${ }^{24}$ Thus, researches of ABCG2 inhibitors have so far failed becoming the "valley of death" in drug development. One of the critical obstacles to discover ABCG2 inhibitors is the lack of extensive understanding of protein-ligand interaction. Currently only an apo crystal structures $(5 \mathrm{NJ} 3)^{25}$ was determined as ABCG2. What's more, there are three regions in ABCG2 that are recognized as the candidate binding sites for inhibitors: (i) specifically targeting the binding site of substrates and the common translocation pathway composed of various sub-sites for multiple scaffolds ${ }^{26}$ (site A); (ii) specifically targeting the nucleotide-binding domains (NBDs) region to interfere with ATP hydrolysis (site B); (iii) specifically targeting the hinge region between NBD and TMD to block the conformational transformation (site C). ${ }^{27}$ In sum, the study of protein (apo)ligand interactions has become more challenging with a wide variety of inhibitors targeting multiple binding sites.

Herein, we have described a comprehensive computational workflow (Fig. 2) to elucidate the protein-ligand interaction of ABCG2 (site A) with the aid of AlphaSpace, ${ }^{28}$ a fragment-centric topographic mapping tool. Its attractive feature is capability to detect the fragment-centric modularity at protein surface and to characterize the large protein binding interface as a set of localized, fragment-targetable interaction pocket. $^{28}$ Firstly, candidate ligands targeting site $\mathrm{A}$ are screened out from inhibitors library (Fig. S1†) according to the rule of spatial complementarity towards the protein surface which was analyzed by AlphaSpace. Secondly, this surface map of protein further guides a suitable binding pose selection from molecular docking output. Finally, classical molecular simulation, which accelerated molecular simulation along with MM/PBSA binding energy calculations are employed to verify the accuracy of

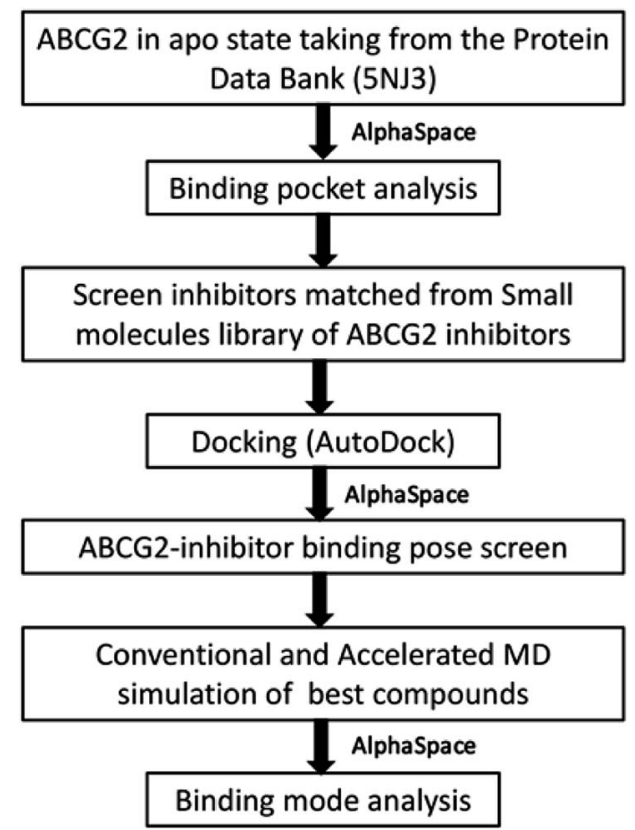

Fig. 2 The flow chart depicting the methodology employed in this study. 
candidate ligands screen and binding pose selection, and to deeply study the interaction of inhibitors with ABCG2 and develop a high-quality pharmacophore model. More importantly, with the understanding of the favourable interactions between ABCG2 protein and inhibitors, we can start to rationalize design novel specific ABCG2 inhibitors by strengthening preferred interactions.

\section{Methods}

\subsection{System preparation}

The initial structures of ABCG2 were taken from Protein Data Bank (PDB) with code $5 \mathrm{NJ} 3 .^{25}$ Gaps in the crystal structure (44$64,300-326,355-369)$ were filled via SWISS-MODEL. ${ }^{29}$ The library consisted of 156 ABCG2 inhibitors were collected from literature and DrugBank (https:/www.drugbank.ca/categories/ DBCAT002662) (Fig S1 $\dagger$ ). The center cavity surface of ABCG2 is characterized with fragment-centric topographical mapping tool, AlphaSpace. ${ }^{28}$ The inhibitors which possible targeted to the center cavity are screened out based on the topographic mapping of center cavity surface and the rule of spatial complementarity. In this study, we select sildenafil, vardenafil (two approved drugs) and E9 as represented compound for further research. Then the selected inhibitors are docked into the active site of ABCG2 via AutoDock Vina ${ }^{30}$ using the standard setting. The binding pose of sildenafil/ABCG2 complex is determined by long-term molecule dynamics simulation. For vardenafil/ABCG2 complex and E9/ABCG2 complex, the binding poses are picked out according to the topographic mapping analysis. The PDB2PQR server was used to estimate protonation states of the protein at constant $\mathrm{pH} 7$ based on $\mathrm{p} K_{\mathrm{a}}$ calculations. ${ }^{31}$

\subsection{Conventional and accelerated MD simulation}

Conventional MD simulation was carried out with pmemd.cuda module of the AMBER 14 package..$^{32}$ Protein models used ff14SB force field, ${ }^{33}$ with TIP3P model. Chloride ions were added to neutralize the system using TIP3P ions with parameters form Joung and Cheatham. ${ }^{34}$ Ligands were parameterized using GAFF force field for the bonded and van der Waals parameters. Partial charges for ligands that fits for the electrostatic potentials were obtained with the use of RESP ${ }^{35}$ which is calculated with Gaussian ${ }^{36}$ at the B3LYP/6-31 $+\mathrm{g}(\mathrm{d}, \mathrm{p})$ level of theory. A cubic periodic box was used with a minimum distance of $10 \AA$ between any box edge as well as any solute atom. All systems were minimized for 10000 cycles of steepest descent followed by 10000 cycles of conjugate gradient. The minimized system was gradually heated from $0 \mathrm{~K}$ to $300 \mathrm{~K}$ under NVT ensemble condition. It equilibrated $3 \mathrm{~ns}$ at $300 \mathrm{~K}$ and followed by $400 \mathrm{~ns}$ of MD simulation under the NPT ensemble condition. The SHAKE algorithm was adopted to bond length constraints. ${ }^{37}$ The time step and non-bonding interaction cut off radius were set to 2 fs and $10 \AA$, respectively.

The aMD protocol ${ }^{38,39}$ which modifies the initial potential energy surface of the biomolecular system was applied to enhance conformational sampling of the systems, and, to enlarge the accessible time scale of cMD. Acceleration comes from a "boost potential", $\Delta V(r)$, was added to the original dihedral potential, $V(r)$, which increases the energy to $V^{*}(r)$ within basins, by using the equations

$$
V^{*}(r)=V(r)+\Delta V(r)
$$

and

$$
\Delta V(r)=\left\{\begin{array}{cc}
0 & V(r) \geq E \\
\frac{(E-V(r))^{2}}{\alpha+E-V(r)} & V(r)<E
\end{array}\right.
$$

where $E$ is a threshold dihedral energy specified by the user. It controls the level of the potential surface affected by bias, and $\alpha$ is the acceleration parameter which establishes the shape of the modified potential.

Thus, it is quite important to choose an appropriate $E$ and $\alpha$ for the transition from one state to another. In this work, value $E$ and $\alpha$ come from the following equation: $E=V_{\mathrm{av}}+V_{\mathrm{av}} \times c$ where $V_{\mathrm{av}}$ is the average dihedral energy from $10 \mathrm{~ns}$ cMD simulation $c$ is a constant and should be specified by the user. Constant 0.2 was chosen to conduct all ABCG2 complex simulations with random velocities.

Saved snapshots were analysed using cpptraj module ${ }^{40}$ in AmberTools 15. The principal component analysis (PCA) was performed with R-based package Bio3 $\mathrm{d}^{41}$ and pharmacophore model was established on the Discovery Studio (DS v4.5). ${ }^{42}$ All figures were produced with Chimera, ${ }^{43}$ Matplotlib $^{44}$ and Microsoft Excel.

\subsection{Protein pocket and binding pocket analysis}

Binding pocket analysis was performed via AlphaSpace, a computational tool for fragment-centric topographical mapping of intermolecular interfaces. ${ }^{28}$ Pockets were selected for analysis based on direct contact with any atom from ligand. The occupation status of individual alpha-space within each pocket was evaluated based on the distance (using $1.6 \AA$ cutoffs) between its associated alpha-atom and the nearest atom from peptide ligand. The total pocket occupation residue was calculated by taking the sum of all occupied alpha-space volumes associated with that residue.

\section{Results and discussion}

\subsection{Screening of candidate inhibitors based on the topographical mapping analysis}

Human ABCG2 protein forms a symmetric homodimer with a two-folded axis perpendicular in the plasma membrane. It is captured in a conformation that is with a central cavity between two transmembrane domains (TMDs) (Fig. 3a). The conformation faces inward and reflects apo state. The central cavity has proven to be the place where the substrate was combined and transported as well as the blocking site for inhibitors. ${ }^{25}$ Thus, we analysed the transmission interface of ABCG2 with AlphaSpace. ${ }^{28}$ The shape of central cavity surface is a typical structure as “凸” (Fig. 3a and b). Its surface is mainly composed of 


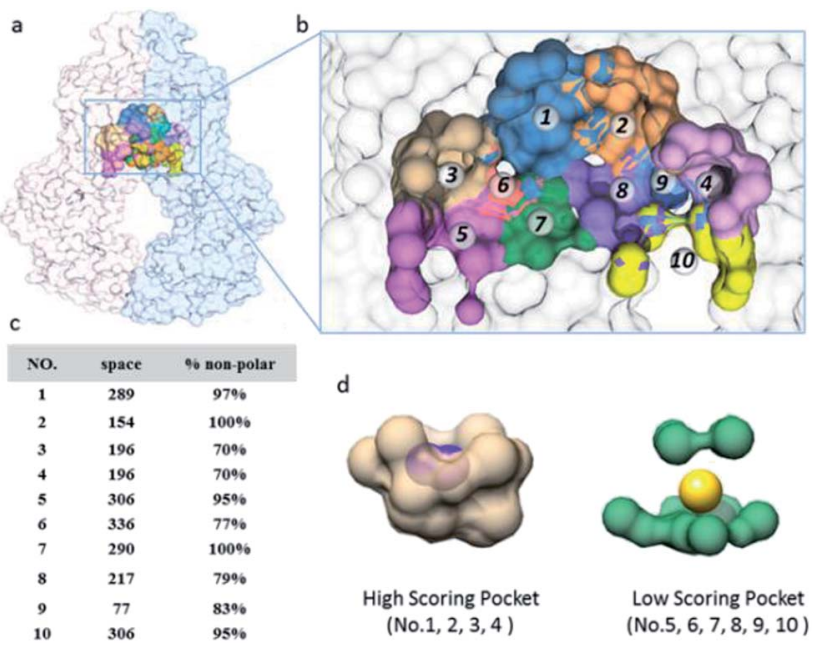

Fig. 3 Surface characterization of ABCG2 channel. (a) Surface representation of $A B C G 2$ structure. The central cavity is shown in colored surfaces. (b) Fragment-centric pocket analysis performed using AlphaSpace. (c) The table presents the results of all pockets, including pocket space $\left(\AA^{3}\right)$, and percentage of non-polar atoms. (d) Representative deep pockets and shallow pockets of ABCG2 channel.

hydrophobic and non-polarized residues (Fig. 3c), which are very consistent with experimental results that the lipophilicity is as a predictor of ABCG2 inhibitors. ${ }^{45}$ As shown in Fig. $3 \mathrm{~b}$ and d, the geometry of sub-pockets can be classified into two categories: deep pocket and shallow pocket (Fig. S2 $\uparrow$ ). Interestingly, the four deep sub-pockets were all located at the edge of cavity thus favourable for ligand binding. It can be speculated that pockets 1-4 are the sites where the inhibitor pharmacophore binds. Correspondingly, the shallow pockets (pockets no. 5-10) make up two planar parts of the cavity. The average height of cavity is $8.08 \AA$ (Fig. $\mathrm{S} 3 \dagger$ ) which is suitable for planar ligand to intrude only. In addition, each plane has a phenylalanine (Fig. S4†) which a $\pi-\pi$ stacking can be easily formed with a planar ligand. Thus, this conformation feature is consistent with QSAR results that planar structure increases the ABCG2inhibitory properties of a drug. We suppose pockets 5-10 are the sites that are suitable for the binding of the inhibitor scaffold.

Based on pocket-centric topographic mapping analysis and the rule of spatial complementarity, the molecular profile of ABCG2 inhibitors that target site A can be easily depicted (as shown in Fig. 4a). In addition, we hypothesized that when the space at the top (pocket 1 and pocket 2) are filled by inhibitors, the conformational changes of TMDs will induce by the motion of NBDs. Thus, the structure of ABCG2 inhibitors must contain two groups that simultaneously bind with two deep pockets at the top. It also needs to bind with a planar moiety and the groups that bind with pocket 3 or pocket 4 are optional (Fig. 4a). After screening of the known inhibitors library (Fig. S1†), 32 compounds were found to meet the above criteria. In this study, sildenafil,${ }^{46}$ vardenafil,${ }^{47}$ E9 (ref. 48 ) (Fig. 4 b) were chosen as candidate inhibitors to explore the protein-ligand interaction at site A.

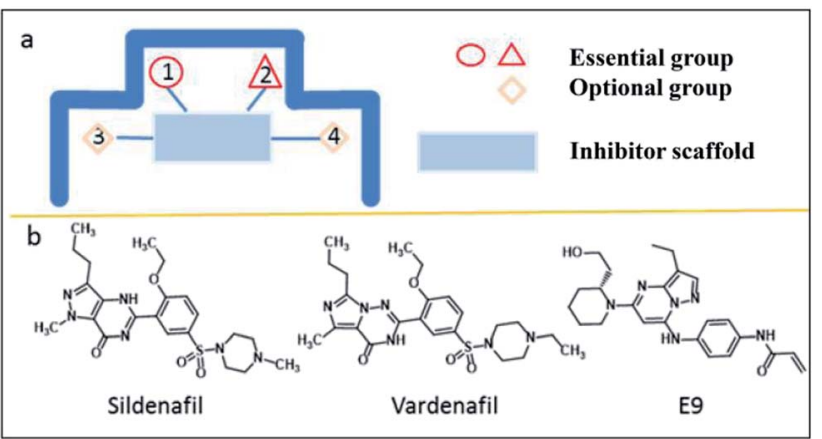

Fig. 4 (a) Structural characteristics of ABCG2 inhibitors. A planar moiety linked by two essential groups and two optional groups. (b) Candidate inhibitors of ABCG2, including sildenafil, vardenafil and E9.

\subsection{Selecting docking pose based on the topographical mapping analysis}

Ranking of docking-generated poses is a challenging problem as sampling algorithms can generate binding poses that is close to the correct solution. At the same time, identifying the nearnative binding poses using scoring functions has been always difficult as well. ${ }^{49,50}$ A reliable method for screening correct binding poses would be able to rank near-native binding poses higher than the poses far away from the native. More importantly, the selected binding poses should be stably combined with proteins and have the capacity to produce biological effects. Sildenafil, vardenafil and E9 were docked into the central cavity of ABCG2 via AutoDock Vina, respectively, and Auto dock energy scoring parameters are listed in Table S1. $\dagger^{30}$ The 20 docking poses of sildenafil were shown in pocket-centric topographical map calculated by AlphaSpace (Fig. 5). The docking poses of vardenafil and E9 are shown in Fig. S5. $\dagger$ From Fig. 5, it can be easily observed that some docking poses are with a high score, such as no. 1, 2, 3. They are not the most appropriate, because of the significant flexibility due to their location. However, sildenafil only stabilized at the top of the central cavity to play a role in blocking substrate efflux. As discussion above, the structure of the ABCG2 inhibitors must contain two essential groups that simultaneously bind to the two deep pockets (Fig. 4). Therefore, pose 15 is the best binding pose of sildenafil against ABCG2. Correspondingly, binding pose 13 for vardenafil and binding pose 10 for E9 (Fig. S5†) were chosen as candidate structures to explore the protein-ligand interaction against $\mathrm{ABCG} 2$ at site $\mathrm{A}$.

In order to verify the accuracy of the docking pose selection method which is fragment-based topographical mapping analysis, molecular dynamics and accelerated molecular dynamics (aMD) of ABCG2/sildenafil complex were employed to track the motion of sildenafil. 20 docking poses of sildenafil were divided into 7 categories based on the RMSD (Fig. S6 $\dagger$ ). After a long-time simulation, two types of movement patterns from seven MD trajectories have been found (Fig. 6). Remarkably, the first type of sildenafil was dramatically and stably bound to the top of central cavity of ABCG2 (trajectory E and trajectory G). This type is consistent with our hypothesis that two groups of sildenafil 

them, the binding pose 15 selected with the help of pocketcentric topographical mapping method belongs to the first type. Thus, the interaction between sildenafil and ABCG2 is so weak that sildenafil could not play the inhibitory effect in these cases.

\subsection{Inhibitors stabilize ABCG2 transport in an inward-facing conformation}

The ATP dependent conformation switching from inwardfacing to outward-facing occurs in ABCG2-mediate substrate transport. $^{51}$ Thus, a long-term lock of transporter in inwardfacing state to prevent ATP binding and hydrolysis is a critical approach to suppress the transport activity of ABCG2. ${ }^{52}$

To explore the stability of ABCG2 when combined with inhibitors, classical molecular dynamics (cMD) were performed to characterize the dynamical behaviour of ABCG2-inhibitors (sildenafil, vardenafil, and E9) complexes. RMSD values of ABCG2 $\mathrm{C} \alpha$ atoms during the $500 \mathrm{~ns}$ production phase that are relative to the initial structures were calculated and plotted in Fig. 7a. The trajectory of the ABCG2/E9 complex fluctuates more than two other trajectories at around 50-80 ns. After that it went parallel to each other, which indicates the conformations of all three ABCG2-inhibitors complexes were in good equilibrium. The result also signifies that conformation of all three complexes were similar to the apo structures during the course of simulations with RMSD values around 2.0 to $2.5 \AA$ to ensure stable trajectories (Fig. S7†).

We further performed accelerated molecular dynamics (aMD) simulation which has the capability of capturing

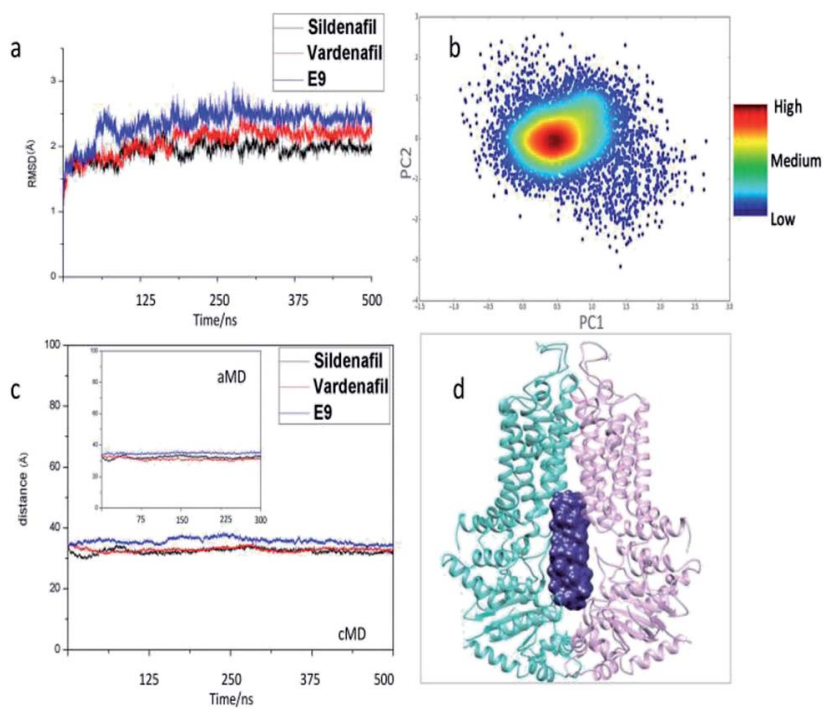

Fig. 7 Inhibitors stabilized the structure of ABCG2 in an inward-facing conformation. (a) The root-mean-square deviation (RMSD) of all atoms in ABCG2 was relative to the crystal structures (PDBID: 5NJ3). (b) Projection of TMDs conformational space simulated by aMD onto the principal components produced from the PCA analysis aMD trajectories. (c) The distance between centres of the two NBDs was measured during the CMD and aMD. (d) A typical structure after MDs represents inhibitors that stabilized ABCG2 protein in an inward-facing conformation. millisecond time scale events to ensure these inhibitors tightly bind to the transport cavity and persistently lock the overall topology structure of ABCG2 in inward-facing conformation. The two-dimensional image of ABCG2/sildenafil trajectories projected to the principal components (PCs) is shown in Fig. 7b. In our $300 \mathrm{~ns}$ aMD simulation, the inward-facing conformation was observed (Fig. 7d), but no other conformations such as occluded conformation and outward-facing conformation were found. In addition, the distance between the centres of two NBDs was measured during cMD and aMD (Fig. 7c and d). The data elucidates that the distances always fluctuate around $33 \AA$, which is close to the apo structure with the distance of $35.3 \AA$.

Taking all the above results together, we come to a conclusion that three inhibitors lock the structure of ABCG2 in inwardfacing state with no significant conformational changes. Therefore, the three small molecules are potential inhibitors of ABCG2 which can effectively block the substrates efflux out. What's more, the MD results suggest that the binding poses selected by fragment-centric topographical mapping method are correct.

\subsection{Understanding the protein-ligand interaction of ABCG2}

Three inhibitors permanently stayed inside the transport cavity throughout the simulation and presented strong binding ability toward ABCG2. The calculated binding interaction of ABCG2/ sildenafil, ABCG2/vardenafil, ABCG2/E9 complexes are -39.70, $-37.63,-46.96 \mathrm{kcal} \mathrm{mol}^{-1}$, respectively. In accordance with the components of the binding interaction from Table $\mathrm{S} 2, \uparrow$ the favourable binding energy is van der Waals interactions $\left(\Delta E_{\mathrm{vdw}}\right)$, the non-polar solvation energy $\left(\Delta G_{\text {nopol }}\right)$ and intermolecular electrostatic interactions $\left(\Delta E_{\text {ele }}\right)$ in all three complexes. The polar solvation energy $\left(\Delta G_{\text {pol }}\right)$ effects weakened inhibitor bindings. The data is consistent with the result of previous pocket analysis that the cavity surface of ABCG2 is mainly composed of hydrophobic residues. It can be speculated that hydrophobicity is a fundamental factor for the design of novel ABCG2 inhibitors.

Our next objective is to understand the protein-ligand interaction of ABCG2 with small molecular inhibitors by means of AlphaSpace matching ${ }^{28}$ and MM-PBSA decomposition analysis. As shown in Fig. 8, the overall shape of the central cavity is basically unchanged, only some sub-pockets have been reorganized after inhibitors were added. For example, P6 was merged into P2 with the space volume increased from 154 to 474 in ABCG2/vardenafil complex (Fig. 8c). Remarkably, by displaying the fragment-centric topographical mapping of each interface, we can easily find out the conserved pockets for binding inhibitors. P1, P2 and P4 were simultaneously occupied by small molecular with the occupied value above $65 \%$ in all three complexes. This is consistent with MM-PBSA decomposition analysis that residues which make up these pockets are primarily involved in the interaction of ABCG2/inhibitors complexes (Fig. 9). It indicates that these sites are crucial for inhibitors binding. Moreover, the three deep sub-pockets were all located at the edge of cavity which particularly facilitates the ligand bind. And the high score pocket P1, P2, P4, P7, P8, and P9 


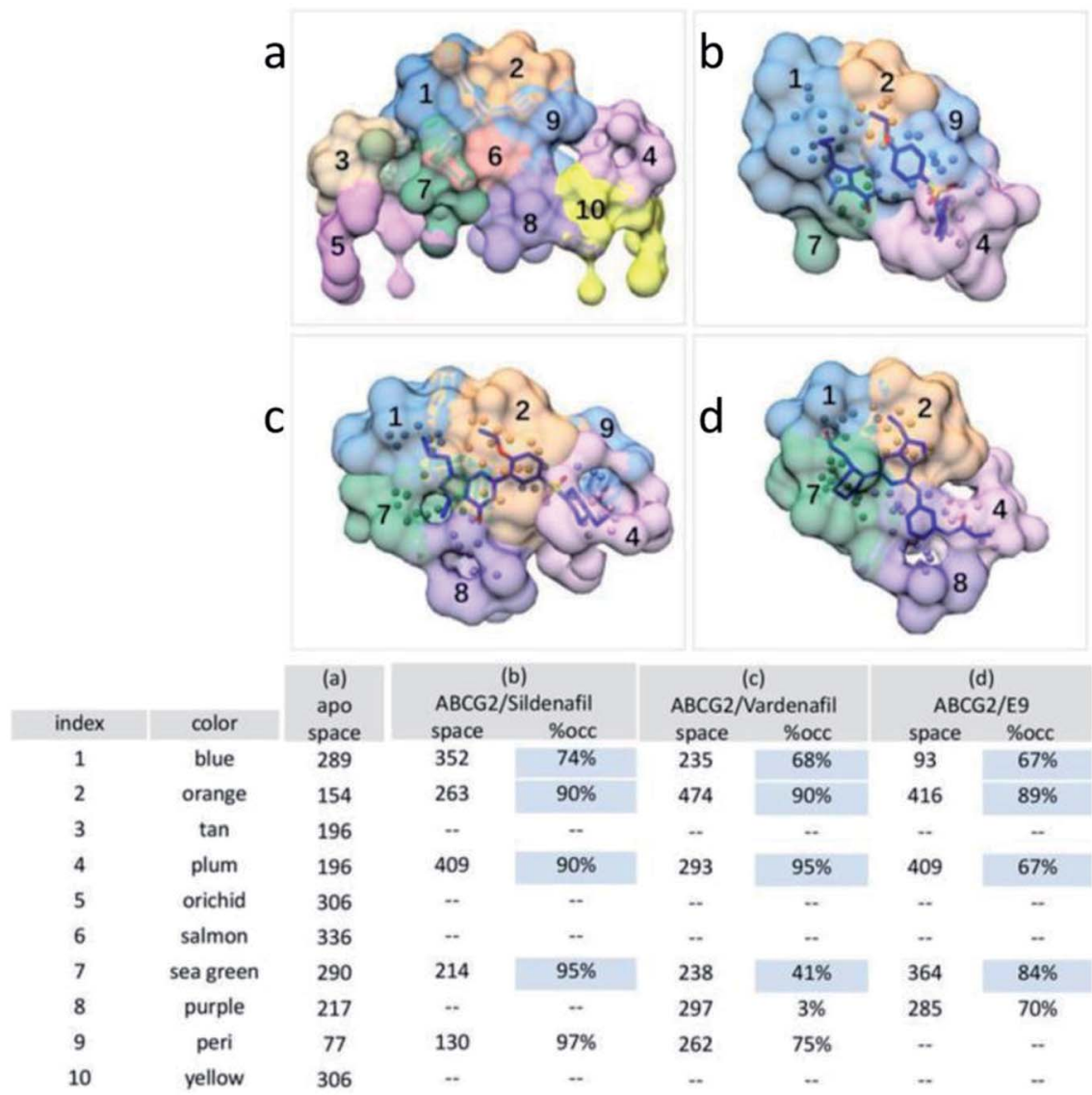

Fig. 8 Pocket matching. (a) Apo, (b) ABCG2/sildenafil, (c) ABCG2/vardenafil, and (d) ABCG2/E9. This table presents the matching results that correspond to for all pockets, including pocket space $\left(\AA^{3}\right)$, and pocket occupation percentage.

are mainly composed by hydrophobic and non-polarized residues (Table S3 $†$ ). For example, at the sildenafil/ABCG2, the hydrophobic residues F432, F439, I543, V546, and M549 have strong interaction with ligand which are very consistent with experimental results and percent of non-polar atoms measured by AlphaSpace (Fig. 3c). And a mutation of high energy residue V546 in ABCG2 to a phenylalanine also interfered with biliary

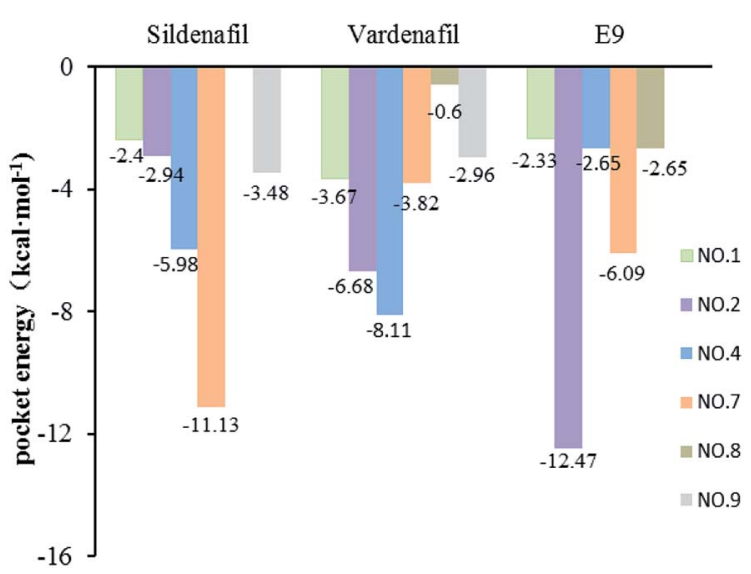

Fig. 9 Decomposition of $\Delta G$ on a per-residue basis for the ABCG2/ inhibitors complexes. cholesterol transport, which was interpreted as a possible steric clash with bound cholesterol by the larger, aromatic residue. Besides the importance of pharmacophores, planar structure from the inhibitors can form a stable sandwich-like structure with residues F439, F439' in the upper and lower plane of cavity, respectively (Fig. S4†). The sandwich-like structure greatly contributed to the high stability of ABCG2-inhibitor interaction with a calculated $\pi-\pi$ stacking energy that is higher than $-3.8 \mathrm{kcal} \mathrm{mol}^{-1}$ in all complexes. Among them, the energy even reached $-11.1 \mathrm{kcal} \mathrm{mol}^{-1}$ in ABCG2/sildenafil complex among them. The result indicates that this planar aromatic group is an important component for ABCG2 inhibitors.

As is known to all that the substrates transported by ABCG2 are driven by the conformational change of TMDs. Thus, pocket 1 and 2 at the top of central cavity will inevitably experience shape transformation during the transport. In this study, we discovered that simultaneously binding to two pockets(P1, P2) can effectively lock ABCG2 protein structure in an inward-facing conformation (Fig. 10a-c). It can be concluded that the groups (site A and B, Fig. 10d) which can bind with pockets 1 and 2 are essential pharmacophores for ABCG2 inhibitors. The second typical structure of inhibitors is the planar aromatic moiety (site C, Fig. 10d). On one hand, it fixes functional groups in the right place. On the other hand, this group forms a $\pi-\pi$ interaction with residue F439 and F439' on the cavity which plays an 

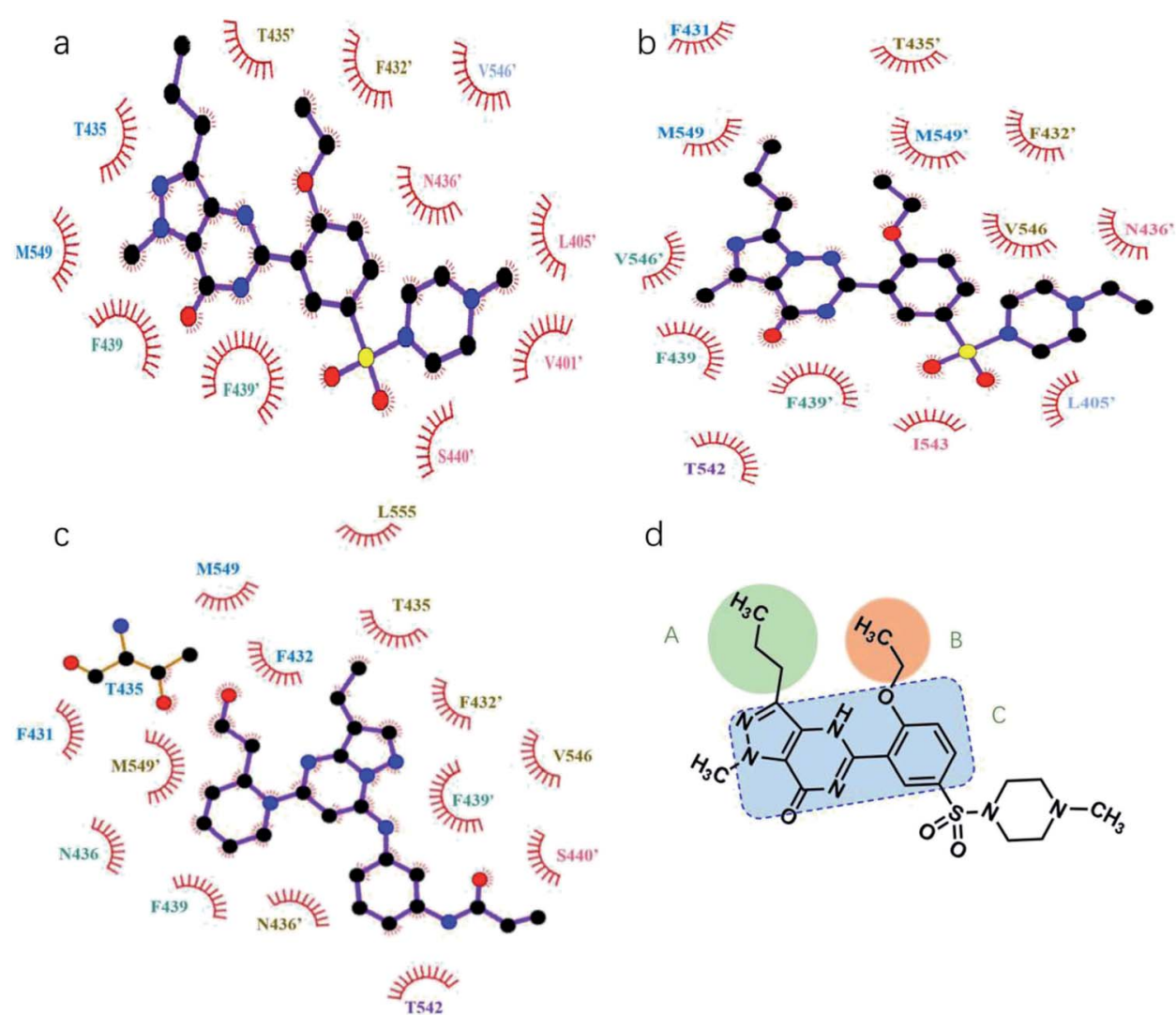

d

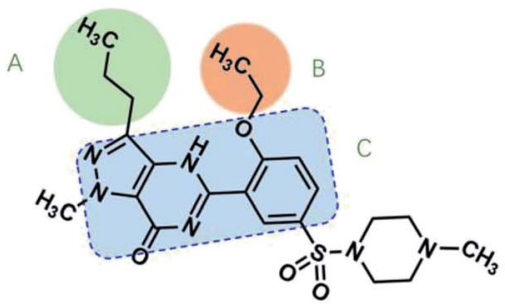

Fig. 10 (a-c). LigPlot scheme for binding ligand residues. The scheme shows the interaction between ABCG2 protein and three inhibitors ((a) sildenafil, (b) vardenafil and (c) E9), respectively. (d) The pharmacophore modelling of ABCG2 inhibitors.

important role in stabilizing complexes. But, the planar aromatic moiety is not a determining factor in distinguishing between inhibitors and substrates for all substrates with aromatic groups. Therefore, it would be a specific scaffold structure for ABCG2 inhibitors.

\section{Conclusions}

The lack of a well resolved binding mode between ABCG2 and ligands is one of the reasons for hindering the discovery of new inhibitors of ABCG2. In the present work, a fragment-centric topographical mapping method ${ }^{28}$ (AlphaSpace software), is adopted to guide the study of molecular mechanism of inhibitor against ABCG2-mediated efflux of drugs. The conventional molecular dynamics and accelerated molecular dynamics simulations have been carried out to understand the interaction of ABCG2/inhibitors. The followings are major findings from the present work.

(1) Fragment-centric topographical mapping method is a powerful tool to guide the study of protein-ligand interaction. It has the capability of detecting the fragment-centric modularity at the protein surface and characterizing large protein binding interface as a set of localized, fragment-targetable interaction pocket. This topographical map of intermolecular interfaces provides great convenience for known inhibitors screening and docking poses selection.
(2) Fragment-centric topographical matching is an efficient method of summarizing the interaction of various inhibitors with receptors. It has the ability of tracking pockets at a fragment-centric resolution and assessing the degree of structural conservation or flexibility at the protein surface among several ABCG2/inhibitors complexes.

(3) Inhibitors simultaneously binding to the two pockets at the top of cavity can effectively lock ABCG2 structure in an inward-facing conformation in suppressing ABCG2 transport.

(4) A credible model for ABCG2 inhibitors was developed. Two groups that simultaneously bound to two pockets (P1, P2) at the top of cavity is the primary and the most important pharmacophoric moiety for ABCG2 inhibitors. And the planar aromatic group linked by pharmacophores is the scaffold of inhibitor which plays key role in stabilizing the interaction of ABCG2/inhibitors complexes (Fig. 10d).

The present study has developed a comprehensive computational strategy to understand the protein-ligand interaction with the help of AlphaSpace, ${ }^{28}$ a fragment-centric topographic mapping tool. We expect that our current studies can provide theoretical aids for designs of high effective drugs targeting ABCG2 to cure diseases such as cancer resistant.

\section{Conflicts of interest}

The authors declare no competing financial interest. 


\section{Acknowledgements}

This work is supported by grants from the Shandong Province independent innovation and transformation of Scientific \& Technological achievements project (No. 2014ZZCX02104), the Primary Research \& Development Plan of Shandong Province (No. 2015GSF118175) and the National Undergraduates' Innovation and Entrepreneurship Training Program (No. 201710445061).

\section{References}

1 P. Anand, D. Nagarajan, S. Mukherjee and N. Chandra, Database, 2014, 2014, bau029.

2 H.-J. Böhm and G. Schneider, Protein, WILEY-VCH Verlag GmbH \& Co. KGaA, Weinheim, 2003.

3 I. Schlichting, Protein-Ligand Interactions: Methods and Applications, ed. G. Ulrich Nienhaus, Humana Press, Totowa, NJ, 2005, pp. 155-165, DOI: 10.1385/1-59259-9125:155.

4 N. Andrusier, E. Mashiach, R. Nussinov and H. J. Wolfson, Proteins, 2008, 73, 271-289.

5 H. Li, K.-S. Leung, M.-H. Wong and P. J. Ballester, $B M C$ Bioinf., 2016, 17, 308.

6 O. Trott and A. J. Olson, J. Comput. Chem., 2010, 31, 455-461.

7 R. A. Friesner, R. B. Murphy, M. P. Repasky, L. L. Frye, J. R. Greenwood, T. A. Halgren, P. C. Sanschagrin and D. T. Mainz, J. Med. Chem., 2006, 49, 6177-6196.

8 H. Li, K.-S. Leung, P. J. Ballester and M.-H. Wong, PLoS One, 2014, 9, e85678.

9 K. Terayama, H. Iwata, M. Araki, Y. Okuno and K. Tsuda, Bioinformatics, 2018, 34, 770-778.

10 M. L. Verdonk, P. N. Mortenson, R. J. Hall, M. J. Hartshorn and C. W. Murray, J. Chem. Inf. Model., 2008, 48, 2214-2225.

11 S.-Y. Huang and X. Zou, Int. J. Mol. Sci., 2010, 11, 3016-3034.

12 K. M. Elokely and R. J. Doerksen, J. Chem. Inf. Model., 2013, 53, 1934-1945.

13 E. M. Leslie, R. G. Deeley and S. P. Cole, Toxicol. Appl. Pharmacol., 2005, 204, 216-237.

14 R. Gatenby and J. Brown, Cold Spring Harbor Perspect. Med., 2017, 8, a033415.

15 P. D. Eckford and F. J. Sharom, Chem. Rev., 2009, 109, 29893011.

16 B. C. Baguley, Mol. Biotechnol., 2010, 46, 308-316.

17 T. Ozben, FEBS Lett., 2006, 580, 2903-2909.

18 K. Natarajan, Y. Xie, M. R. Baer and D. D. Ross, Biochem. Pharmacol., 2012, 83, 1084-1103.

19 R. Allikmets, L. M. Schriml, A. Hutchinson, V. Romano-Spica and M. Dean, Cancer Res., 1998, 58, 5337-5339.

20 L. A. Doyle, W. Yang, L. V. Abruzzo, T. Krogmann, Y. Gao, A. K. Rishi and D. D. Ross, Proc. Natl. Acad. Sci. U. S. A., 1998, 95, 15665-15670.

21 M. Dean, A. Rzhetsky and R. Allikmets, Genome Res., 2001, 11, 1156-1166.

22 W. Mo and J.-T. Zhang, Int. J. Biochem. Mol. Biol., 2012, 3, 127.
23 S. K. Rabindran, H. He, M. Singh, E. Brown, K. I. Collins, T. Annable and L. M. Greenberger, Cancer Res., 1998, 58, 5850-5858.

24 J. Ricci, D. Lovato and R. Larson, J. Dev. Drugs, 2015, 4, 138. 25 N. M. I. Taylor, I. Manolaridis, S. M. Jackson, J. Kowal, H. Stahlberg and K. P. Locher, Nature, 2017, 546, 504.

26 S. M. Jackson, Nat. Struct. Mol. Biol., 2018, 25(4), 333.

27 M. K. Krapf, J. Gallus, V. Namasivayam and M. Wiese, J. Med. Chem., 2018, 61, 7952-7976.

28 D. Rooklin, C. Wang, J. Katigbak, P. S. Arora and Y. Zhang, J. Chem. Inf. Model., 2015, 55, 1585-1599.

29 F. Kiefer, K. Arnold, M. Künzli, L. Bordoli and T. Schwede, Nucleic Acids Res., 2008, 37, D387-D392.

30 W. L. Jorgensen, Science, 2004, 303, 1813-1818.

31 T. J. Dolinsky, P. Czodrowski, H. Li, J. E. Nielsen, J. H. Jensen, G. Klebe and N. A. Baker, Nucleic Acids Res., 2007, 35, W522-W525.

32 D. Case, V. Babin, J. Berryman, R. Betz, Q. Cai, D. Cerutti, T. Cheatham III, T. Darden, R. Duke and H. Gohlke, Amber 14, University of California, San Francisco, 2014.

33 J. A. Maier, C. Martinez, K. Kasavajhala, L. Wickstrom, K. E. Hauser and C. Simmerling, J. Chem. Theory Comput., 2015, 11, 3696-3713.

34 W. L. Jorgensen, J. Chandrasekhar, J. D. Madura, R. W. Impey and M. L. Klein, J. Chem. Phys., 1983, 79, 926935.

35 C. I. Bayly, P. Cieplak, W. Cornell and P. A. Kollman, J. Chem. Phys., 1993, 97, 10269-10280.

36 M. J. Frisch, G. W. Trucks, H. B. Schlegel, G. E. Scuseria, M. A. Robb, J. R. Cheeseman, G. Scalmani, V. Barone, B. Mennucci, G. A. Petersson, H. Nakatsuji, M. Caricato, X. Li, H. P. Hratchian, A. F. Izmaylov, J. Bloino, G. Zheng, J. L. Sonnenberg, M. Hada, M. Ehara, K. Toyota, R. Fukuda, J. Hasegawa, M. Ishida, T. Nakajima, Y. Honda, O. Kitao, H. Nakai, T. Vreven, J. A. Montgomery, J. E. Peralta, F. Ogliaro, M. Bearpark, J. J. Heyd, E. Brothers, K. N. Kudin, V. N. Staroverov, R. Kobayashi, J. Normand, K. Raghavachari, A. Rendell, J. C. Burant, S. S. Iyengar, J. Tomasi, M. Cossi, N. Rega, J. M. Millam, M. Klene, J. E. Knox, J. B. Cross, V. Bakken, C. Adamo, J. Jaramillo, R. Gomperts, R. E. Stratmann, O. Yazyev, A. J. Austin, R. Cammi, C. Pomelli, J. W. Ochterski, R. L. Martin, K. Morokuma, V. G. Zakrzewski, G. A. Voth, P. Salvador, J. J. Dannenberg, S. Dapprich, A. D. Daniels, Ö. Farkas, J. B. Foresman, J. V. Ortiz, J. Cioslowski and D. J. Fox, Gaussian 09, Revision D.01, Gaussian Inc., Wallingford, CT, 2009.

37 T. G. Coleman, H. C. Mesick and R. L. Darby, Ann. Biomed. Eng., 1977, 5, 322-328.

38 D. Hamelberg, J. Mongan and J. A. McCammon, J. Chem. Phys., 2004, 120, 11919-11929.

39 D. Hamelberg, C. A. de Oliveira and J. A. McCammon, J. Chem. Phys., 2007, 127, 155102.

40 D. R. Roe and T. E. Cheatham, J. Chem. Theory Comput., 2013, 9, 3084-3095.

41 B. J. Grant, A. P. C. Rodrigues, K. M. ElSawy, J. A. McCammon and L. S. D. Caves, Bioinformatics, 2006, 22, 2695-2696. 
42 D. Studio, Release 4.5, Accelrys Software Inc., 2016.

43 E. F. Pettersen, T. D. Goddard, C. C. Huang, G. S. Couch, D. M. Greenblatt, E. C. Meng and T. E. Ferrin, J. Comput. Chem., 2004, 25, 1605-1612.

44 J. D. Hunter, Comput. Sci. Eng., 2007, 9, 90-95.

45 A. E. Stacy, P. J. Jansson and D. R. Richardson, Mol. Pharmacol., 2013, 84, 655-669.

46 Z. Shi, A. K. Tiwari, S. Shukla, R. W. Robey, S. Singh, I.-W. Kim, S. E. Bates, X.-X. Peng, I. Abraham and S. V. Ambudkar, Cancer Res., 2011, 10, 3820.

47 J. J. Chen, Y. L. Sun, A. K. Tiwari, Z. J. Xiao, K. Sodani, D. H. Yang, S. G. Vispute, W. Q. Jiang, S. D. Chen and Z. S. Chen, Cancer Sci., 2012, 103, 1531-1537.
48 Y. Gao, T. Zhang, H. Terai, S. B. Ficarro, N. Kwiatkowski, M.-F. Hao, B. Sharma, C. L. Christensen, E. Chipumuro, K.-k. Wong, J. A. Marto, P. S. Hammerman, N. S. Gray and R. E. George, Cell Chem. Biol., 2018, 25, 135-142.

49 E. Yuriev, M. Agostino and P. A. Ramsland, J. Mol. Recognit., 2011, 24, 149-164.

50 E. Yuriev and P. A. Ramsland, J. Mol. Recognit., 2013, 26, 215239.

51 D. C. Rees, E. Johnson and O. Lewinson, Nat. Rev. Mol. Cell Biol., 2009, 10, 218.

52 J. A. Coleman and E. Gouaux, Nat. Rev. Mol. Cell Biol., 2018, 25, 170-175. 